\title{
The Benefits of Mindfulness Meditation: Changes in Emotional States of Depression, Anxiety, and Stress
}

\author{
Istvan Schreiner and James P. Malcolm \\ University of Western Sydney, Australia
}

\begin{abstract}
The research investigated the effect of mindfulness meditation on the emotional states of depression, anxiety, and stress. Additionally, we explored whether people with varying severity of depression, anxiety, and stress responded differently to mindfulness training. In order to investigate these questions, participants $(n=50)$ completed the Depression Anxiety and Stress Scale, 21-item version (DASS-21) before and after a 10-week mindfulness meditation program. As predicted, the severity levels of all affective measures have decreased by the end of the meditation course. Participants with severe emotional difficulties at the time of commencing the meditation course demonstrated the most notable improvement over time. These results suggest that mindfulness training is beneficial in reducing the symptoms of subclinical depression and anxiety and can substantially reduce stress. Attentional retraining and self-management aspects of mindfulness meditation, as well as relevant methodological issues are discussed.
\end{abstract}

Keywords: mindfulness, meditation, depression, anxiety, stress

$\mathrm{P}$ sychological interventions based on mindfulness skills are an increasingly popular means of treating various psychological disorders (Baer, 2003; Hayes, Strosahl, \& Wilson, 1999; Salsman \& Linehan, 2006). Developing mindfulness involves purposefully directing one's attention to internal and external experiences, occurring on a moment-to-moment basis, without evaluating or judging these experiences. When practised correctly, mindfulness can aid the development of cognitive skills that are often associated with acceptance, objectivity, and meta-cognition (Baer, 2003; Brown \& Ryan, 2003; Hayes \& Kelly, 2003).

The reported benefits of mindfulness training are numerous. Empirical findings suggest that mindfulness training can be applied for a broad range of life difficulties, ranging from the treatment of depression (Broderick, 2005) and somatic complaints (Sharma, Mishra, \& Balodhi, 1990), to pain management programs (Astin, 2004). Yet, despite the widespread use of mindfulness training in clinical practice, research on the topic is limited. The current research explored whether severity levels of depression, anxiety, and stress were differently influenced by mindfulness meditation.

Mindfulness meditation has been shown to reduce the symptoms of anxiety (Bögels, 2006; Mogg \& Bradley, 2005), depression (Broderick, 2005; Teasdale, Segal, \& Williams, 1995), and stress (Grossmana, Niemannb, Schmidtc, \& Walach, 2004; Miller, Fletcher, \& Kabat-Zinn, 1995). Typically, clients are taught to 'observe' their

Address for correspondence: Dr James P. Malcolm, School of Psychology, University of Western Sydney, Locked Bag 1797, Penrith South DC, NSW 1797, Australia.Email: j.malcolm@uws.edu.au 
cognitions, emotions or sensations nonjudgmentally, and to accept them as they are. Teasdale (1999) referred to this ability as 'metacognitive insight', or a decentered view of one's thoughts and emotions. To enhance clients' ability to nonjudgmentally attend to thoughts and emotions, they are trained to utilise various metaphors, such as 'watching clouds passing by' or 'observing a train station with trains arriving and leaving' (Baer, 2003). By also teaching clients to return the focus of attention to a neutral stimuli (e.g., breathing), they learn to regulate attention on a moment to moment basis, undisturbed by maladaptive thought processes.

\section{Mindfulness Training and Depression}

The success of using meditation practice to aid the treatment of depression is achieved by reducing ruminative tendencies and simultaneously increasing attentional control (Ramel, Philippe, Carmona, \& McQuaid, 2004). Dysfunctional attitudes and negative self referent thoughts may be viewed as the content of the mind (Beck \& Clark, 1988), whereas rumination refers to the processes of the mind or how individuals relate to the content (Hayes \& Kelly, 2003; Nolen-Hoeksema, 1991; Nolen-Hoeksema, 2000). When depression prone individuals engage in rumination they engage in a process that 'recycles' the negative thought content and consequently increase the chances of relapse and the severity of the depressive episode (Nolen-Hoeksema, 1991).

Meditation practice for depressed individuals may work on two levels: one, by increasing awareness of the present moment, depressed clients learn to shift their attention from recycling negative self referent information to objectively attending to and processing all available information; and two, by accepting emotions nonjudgmentally people learn to reduce their role in maintaining dysfunctional cognitive schemas (Baer, 2003; Hayes \& Kelly, 2003).

In a recent clinical trial, Mindfulness Based Cognitive Therapy (MBCT; Segal, Williams, \& Teasdale, 2002) effectively decreased depressive symptoms and prevented later relapse (Ma \& Teasdale, 2004). While not all depressed clients benefited equally from MBCT, the majority with up to two previous episodes showed lasting improvement. However, people with more than two episodes of depression, primarily associated with negative life events, may be resistant to the full benefits meditation training.

\section{Mindfulness Training and Anxiety}

Meditation is widely used in the treatment of various anxiety disorders. The rationale for using meditation as a form of treatment is mostly driven by the attentional and arousal theories of anxiety. Generally, anxious individuals scan the environment for threat-related information and interpret ambiguous information as threatening (Bögels \& Mansell, 2004; Hunt, Keogh, \& French, 2006). In addition, people with severe anxiety disorders suffer from chronic over arousal, increasing the likelihood of misinterpreting ambiguous environmental information as threatening (Barlow, 1991).

Similarly to the treatment of depression, people in meditation-based intervention programs for anxiety are trained to nonjudgmentally 'observe' their thoughts and physical sensations. The key component here is accepting and objectively examining physiological symptoms. Although the examination of physiological symptoms is inherently similar to CBT, cognitive therapy trains people to recognise 
the relationship between arousal and cognition (Barlow, 1991). In contrast, mindfulness training draws no direct connection between physical sensations and thoughts, rather aims to teach people to attend to thoughts and to physical sensations as they occur (Kabat-Zin, 1990). Despite directly not targeting over-arousal, meditation alone leads to a significant reduction in muscle tension and to the reduction of overall physiological symptoms of anxiety (Marks, 2000; Mogg \& Bradley, 2005).

\section{Mindfulness Training and Stress Reduction}

The most often associated intervention modality with mindfulness training is related to stress reduction. Stress as a construct can refer to various physiological and cognitive states, including irritability, excessive worry, or over-arousal (Lovibond \& Lovibond, 1995). Chronic stress may also be associated with occupations (Shapiro, Astin, Bishop, Scott, \& Cordova, 2005), terminal illness (Speca, Carlson, Goodey, \& Angen, 2000), or the presence of psychological disorders (Bishop, 2002). While relatively recently developed, Mindfulness Based Stress Reduction programs (MBSR) have been successfully applied in various settings and a number of outcome studies report good results (Kabat-Zinn, 2003).

Originally MBSR was designed to treat chronic pain in medical settings (KabatZinn, 1990). Delivery of the program is group-based, generally consisting of 20 to 30 people. It is usually conducted as an 8- or 10-week course with 2 hour to 2.5 hour sessions, blending discussions about stress reactions, instructions about between session activities, and active meditation skills training. Apart from the traditional mindfulness skills, MBSR also includes a 45 minute exercise of 'body scan', where individuals are taught to direct their attention to body parts and observe the sensations in each area. Although the 'body scan' bears some resemblance to progressive muscle relaxation technique (PMR), no conscious control of muscles is involved; rather the focus is directed to the naturally occurring sensations. The rationale behind the 'body scan' technique is based on the notion that individuals with high arousal and stress levels will likely experience physiological symptoms (such as muscle tension), and attending to these symptoms while maintaining a nonjudgmental frame of mind will reduce these physical responses (Kabat-Zinn, 1990).

From a cognitive perspective, meditation as a stress reduction technique works similarly to the treatment of depression and anxiety. Judgmental thoughts, such as those about the source of stress, are observed as long as they are present, then attention is directed back to the present (Kabat-Zinn, 1990; Kristeller and Hallet, 1999). The results are twofold: one, the regular practice of meditation leads to cognitive change; and two, individuals learn adaptive self management skills. Since mindfulness training promotes the awareness of all emotional and cognitive events as they occur in the present, individuals can recognise the warning signs of tension build up. Once they have acquired these skills of 'metacognitive insight', controlling previously difficult safety behaviours and cognitions become more manageable (Kristeller and Hallet, 1999).

\section{The Current Study}

By integrating the theories of mindfulness training, the present research had multiple aims. First, our main goal was to replicate earlier findings, and consistently we expected to find a notable reduction in individual levels of depression, anxiety and 
stress after attending a 10-week meditation course. Second, we predicted that individuals with depression, anxiety, and stress will respond differently to meditation practice. We predicted comparable reduction in anxiety and stress levels, but a somewhat less pronounced reduction in depression levels. Finally, we hypothesized that individuals with higher levels of stress and anxiety levels will respond more favorably to meditation practice.

\section{Method}

\section{Participants}

All participants were selected from the general public, attending structured 10-week meditation courses at two Well-Aware-Ness meditation centers, located in Sydney, Australia. Data was collected between July, 2006 and September, 2006. All participants were approached at the beginning of the meditation courses and received an information letter about the study. Participation was voluntary and no incentives were offered for partaking.

\section{Time 1}

Participants were 50 volunteers attending meditation programs at two meditation centers. The average age of the participants was 46 years $(S D=17)$. The gender composition of the sample was 32 females and 18 males. The majority of participants were from English speaking backgrounds (94\%) and held professional (46\%) or associate professional (16\%) positions.

\section{Time 2}

During the course of the study three participants dropped out of the meditation programs and a further four participants were not present at data collection at Time 2 and could not be contacted at a later date. Overall, 43 participants completed the questionnaires at both Time 1 and Time 2 .

\section{Measures}

The tests used in the present study were the Depression, Anxiety and Stress Scale - 21 Items (DASS-21; Lovibond \& Lovibond, 1995) and the Reading the Mind in the Eye Test (Baron-Cohen \& Jolliffe, 1997).

\section{The Depression, Anxiety and Stress Scale - 21 Items (DASS-21)}

The DASS-21 (Lovibond \& Lovibond, 1995) is a 21-item self-report measure of negative affect, developed with the specific aim of achieving maximal differentiation between the three syndromes of depression, anxiety and tension/stress. The factor structure of the DASS-21 is stable, and the three scales show good convergent and discriminant validity and high internal consistency both in clinical and in nonclinical samples (Lovibond \& Lovibond, 1995).

\section{Reading the Mind in the Eye Test ('Eyes Test')}

To control for regression artifacts between Time 1 and Time 2 the Reading the Mind in the Eyes Test (Eyes Test) was selected (Baron-Cohen \& Jolliffe, 1997). The Eyes Test is series of 36 black and white photographs of the eye-region of the faces of different actors and actresses, showing different emotions. Test takers are asked to 
choose which of four words best describes what the person in the photograph is thinking or feeling.

The Eyes Test was designed to assess how well people can recognise others' mental states and emotions. The test has good criterion validity and provides a stable measure over time (Baron-Cohen \& Jolliffe, 1997).

\section{Design}

The study was designed to assess the effect of mindfulness meditation on the emotional states of depression, anxiety, and stress. Hence, the design implemented three identical repeated measures ANOVA's for depression, anxiety, and stress, using the DASS-21 scores as between-group variables (normal vs. moderate vs. severe), and passage of time as the within subject variables (Time 1 vs. Time 2 ). To control for statistical artifacts of regression, measures on the Eyes Test between Time 1 and Time 2 were used as covariates.

\section{Procedure}

\section{Time 1}

Prospective participants were approached at the time of commencing the meditation courses where the overall aim of the study was described and a detailed description was provided. Upon agreement, informed consent and demographic information was collected and the two questionnaires were administered (DASS-21, Eyes Test). Completion of the questionnaire packages took approximately 10 to 15 minutes. No additional procedures were implemented.

\section{Meditation Classes}

All meditation classes were conducted and supervised by the same registered psychologist/meditation teacher with extensive experience in therapeutic interventions and in mindfulness meditation teaching. The 10 -week meditation courses consisted of weekly 2 -hour classes focusing on a theme of 'meditation as a form of relaxation' for the duration of the program.

\section{Meditation Class Structures}

The class structures within the meditation courses followed the same routines. For each class, a discussion topic was set and was introduced by the meditation teacher. The introduction lasted approximately 30 minutes, followed by a 30 minute meditation session. Individual experiences and thoughts concerning the main topic and difficulties were discussed after the meditation session. All participants were encouraged to meditate at home privately between the weekly sessions.

Common to all sessions, five core components of meditative practice were emphasised: (a) present centered awareness, (b) attention training, (c) objective, nonjudgmental, nonreactive observation, (d) open receptiveness, and (e) impersonal awareness. The active meditation component within each session was $30 \mathrm{~min}$ utes, thus all participants received the same strategic intervention.

\section{Time 2}

Data collection took place over 2 consecutive weeks, on week 9 and week 10 of the scheduled meditation courses. On week 9 a reminder was given about the forthcoming data collection and participants were asked to attend the final session of the 
Severity Ratings for the DASS-21 and for the Present Study

\begin{tabular}{lccc} 
& Depression & Anxiety & Stress \\
\hline DASS-21 & $0-9$ & & \\
Normal & $10-13$ & $0-7$ & $0-14$ \\
Mild & $14-20$ & $8-9$ & $15-18$ \\
Moderate & $21-27$ & $10-14$ & $19-25$ \\
Severe & $28+$ & $15-19$ & $26-33$ \\
Extremely severe & & $20+$ & $34+$ \\
Present Study & $0-9$ & $0-7$ & $0-14$ \\
$\quad$ Normal* & $10-20$ & $8-14$ & $15-25$ \\
Moderate* & $21+$ & $15+$ & $26+$ \\
Severe+ $^{* *}$ & & & \\
\hline
\end{tabular}

Note: *Normal levels were retained; **DASS-21 Mild and Moderate levels collapsed; ***DASS-21 Severe and Extremely Severe levels collapsed.

courses. Data from five participants was collected with one week delay. In addition, staff at the meditation centre were interviewed about attendance rates. Attendance was regular and overall rates were estimated at $70 \%$ to $80 \%$.

\section{Coding}

Due to the nonclinical sample, coding was initially intended to represent severity ratings based on the extent of score dispersion on the DASS-21. However, the collected data revealed a large enough dispersion that allowed us to follow the recommended diagnostic guidelines (Lovibond \& Lovibond, 1995) more closely. Consequently, the groups consisted of Normal, Moderate, and Severe+ depression, anxiety, and stress conditions. For the recommended and collapsed cut-off scores, see Table 1.

\section{Results}

\section{Eyes Test}

To examine whether our intended covariate showed a stable performance, a paired sample $t$ test compared Time 1 and Time 2 measures on the Eyes Test. As predicted, no overall difference was found between Time $1(M=26.10)$ and Time $2(M=$ 26.50), $t(42)=-.808, p=.424$. In addition, Pearson bivariate correlational analyses were carried out between the Eyes Test results and the DASS-21 measures. Performance on the Eyes Test remained independent from the emotional states of depression, anxiety, and stress. Hence, to control for regression artifacts, the Eyes Test results were incorporated into further analysis as a covariate.

\section{Affective Symptoms on the DASS-2I}

To examine how the groups with different levels of depression, anxiety, and stress were influenced by the meditation courses, we applied repeated measures ANOVAs for each affective state. Severity levels were used as between-group factors and passage of time as within-subject factors. To calculate effect sizes between means, the following formula was implemented: $d=M_{1}-M_{2} / \sigma_{\text {pooled }}$, where $\sigma_{\text {pooled }}=\sqrt{ }\left[\left(\sigma_{1}^{2}+\sigma_{2}^{2}\right) / 2\right]$. Overall, the results showed significant differences for all three measures. 


\section{Depression}

Repeated measures ANOVA for the depression scores revealed a significant main effect for Time, $F(1)=8.31, p<.05$. Depression levels significantly decreased from Time 1 to Time 2 . We also observed a significant interaction effect between the levels of depression across the passage of time, $F(2)=18.30, p<.001$ (Figure 1).

To further analyse the nature of the interaction, $t$ tests were carried out between the relevant mean values (see Table 2). As expected, normal levels of depression scores remained stable over time and no increase or decrease was observed. Similarly, no difference was detected for moderate depression. The only significant difference we observed was within the severe category. Severely depressed participants reported a significant decrease in their depression by the end of the 10-week meditation course, $t(14)=3.39, p=.004$. The obtained effect size for this difference was also large (Cohen's $d=1.70$ ).

\section{Anxiety}

Repeated measures ANOVA for anxiety scores showed a significant main effect for Time, $F(1)=4.11, p=.049$. Anxiety levels were significantly reduced by the end of the meditation course. Also, a significant interaction effect emerged between the levels of anxiety ratings across time, $F(2)=18.49, p<.001$ (see Figure 2).

Upon further analysis, we found no difference for normal anxiety levels. Although the responses between Time 1 and Time 2 showed an increasing trend, the difference did not approach statistical significance. Moderate anxiety levels significantly decreased from Time 1 to Time $2, t(32)=2.35, p=.025$. The effect size for this difference was large (Cohen's $d=0.81$ ). Anxiety level for the severely anxious group dropped significantly by the end of the meditation course, $t(18)=4.54, p<.001$ (see Table 3).

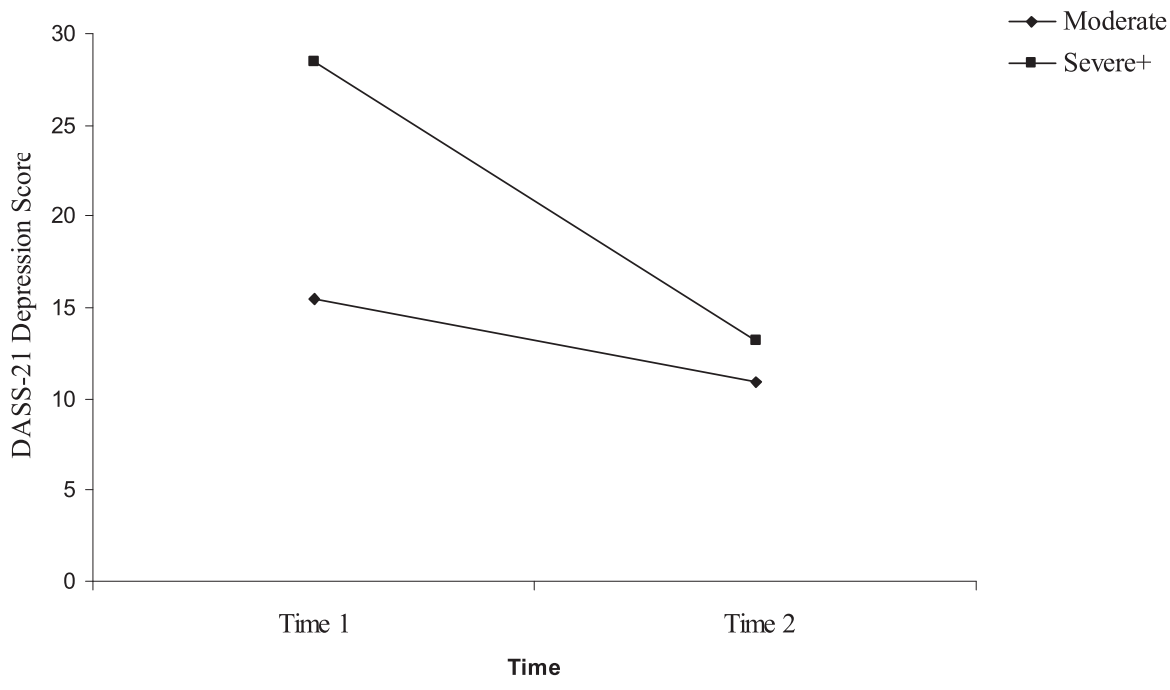

\section{FIGURE 1}

Severity levels for the DASS-21 depression scores at Time 1 and Time $2(n=43)$. 
TABLE 2

Affective Symptoms for Depression at Time 1 and Time $2(n=43)$

\begin{tabular}{|c|c|c|c|c|c|c|}
\hline \multirow[b]{3}{*}{ DASS-21 - Depression } & \multicolumn{6}{|c|}{ Time } \\
\hline & \multicolumn{3}{|c|}{ Time 1} & \multicolumn{3}{|c|}{ Time 2} \\
\hline & $M$ & $S D$ & $N$ & $M$ & $S D$ & $N$ \\
\hline Normal & 4.0 & 2.9 & 26 & 3.0 & 4.3 & 26 \\
\hline Moderate & 15.5 & 4.4 & 9 & 10.9 & 8.4 & 9 \\
\hline Severe+ & 28.5 & 6.9 & 8 & $13.2^{*}$ & 10.7 & 8 \\
\hline
\end{tabular}

Note: ${ }^{*} p<.005$.

\section{Stress}

Repeated measures ANOVA for stress scores revealed a significant main effect across Time, $F(1)=5.66, p<.05$. These results indicate that the overall stress levels significantly decreased by the end of the 10 -week meditation programs. A significant interaction effect was detected between stress levels across time, $F(2)=19.76$, $p<.001$ (see Figure 3).

To further examine the group differences, $t$ tests were carried out (see Table 4). Consistent with our hypothesis, no significant difference was observed for normal stress levels. Participants with moderate levels of stress at Time 1 reported a significant decrease in stress at the end of the meditation courses, $t(26)=2.92, p=.007$. This effect size was large (Cohen's $d=1.04$ ), and indicated approximately $45 \%$ overlap in mean stress scores between Time 1 and Time 2. Stress levels for the severe group also showed a significant decrease by the end of the meditation courses, $t(20)=6.83, p<.001$.

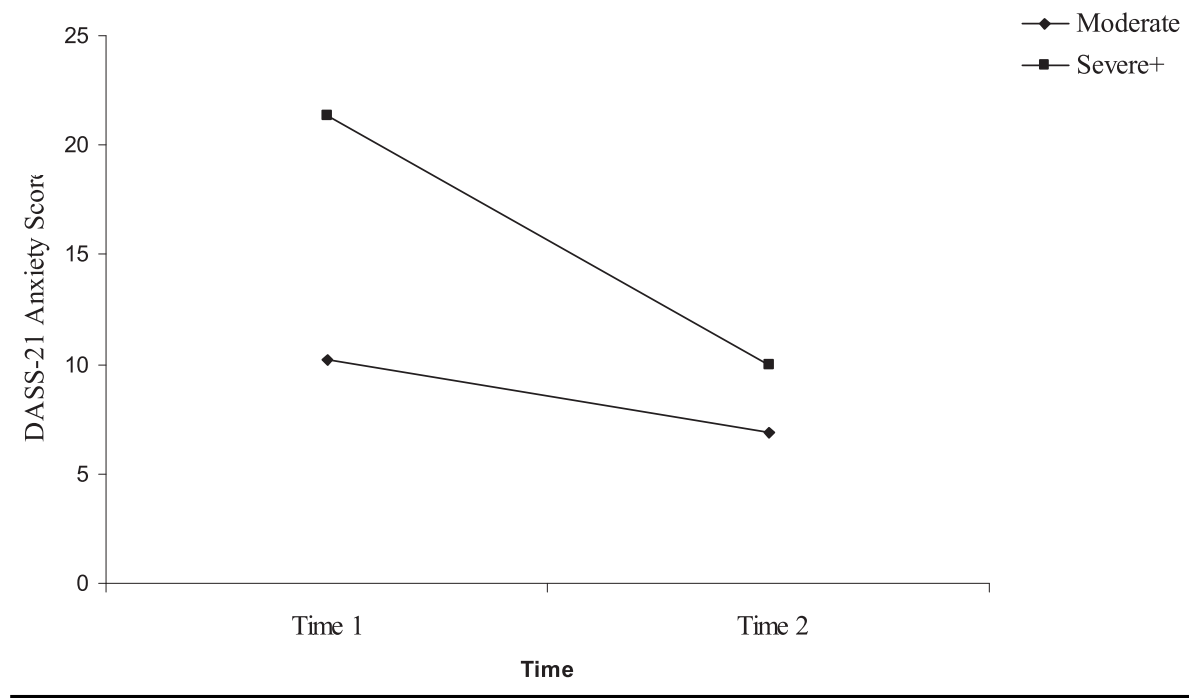

FIGURE 2

Severity levels for the DASS-21 anxiety scores at Time 1 and Time $2(n=43)$. 


\section{TABLE 3}

Affective Symptoms for Anxiety at Time 1 and Time $2(n=43)$

\begin{tabular}{lrcccccc}
\hline & \multicolumn{9}{c}{ Time } \\
\cline { 2 - 8 } DASS-21 - Anxiety & $M$ & $S D$ & $N$ & $M$ & $S D$ & $N$ \\
\cline { 2 - 8 } & 2.4 & 2.4 & 16 & 3.9 & 3.9 & 16 \\
Normal & 10.2 & 3.3 & 17 & $6.9^{*}$ & 4.7 & 17 \\
Moderate & 21.4 & 6.5 & 10 & $10.0^{* *}$ & 4.5 & 10 \\
Severe+ & & & & & & & \\
\hline
\end{tabular}

Note: ${ }^{*} p<.05$ and ${ }^{* *} p<.001$.

\section{Discussion}

The present study investigated the influence of a 10-week mindfulness meditation course on the affective states of depression, anxiety, and stress. To explore this question, 50 members of the general public were asked to complete the DASS-21 before and after attending a 10-week meditation course. We predicted that after the completion of the course all affective measures will decrease in severity. Also, we explored whether people with different levels of depression, anxiety, and stress responded differently to mindfulness meditation. This part of the study was exploratory; however, we predicted that individuals with higher levels of depression, anxiety, and stress will benefit more from mindfulness training.

The results of the study can be summarised into two main findings. First, our main hypothesis regarding affective states and mindfulness meditation was supported. Severity levels of all three affective states decreased significantly by the end of the meditation course. Second, with the exception of moderate depression measures, our results showed that all severity levels decreased after the meditation

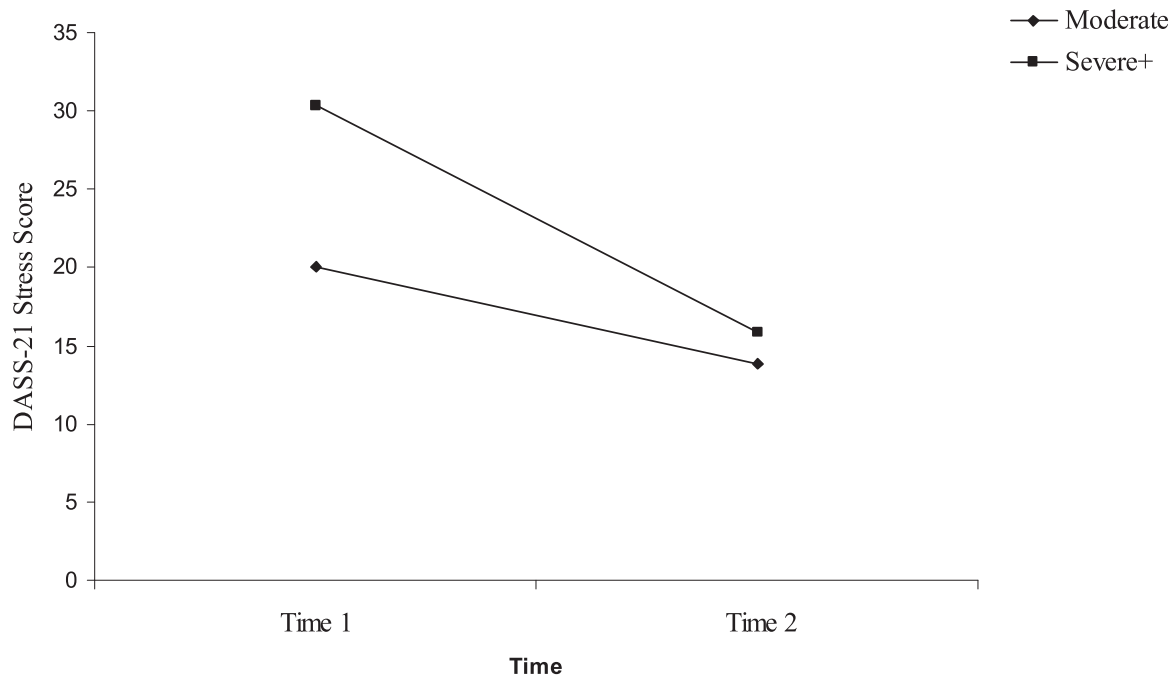

FIGURE 3

Severity levels for the DASS-21 stress scores at Time 1 and Time $2(n=43)$. 
Affective Symptoms for Stress at Time 1 and Time $2(n=43)$

\begin{tabular}{lrrrrrrr}
\hline & \multicolumn{9}{c}{ Time } \\
\cline { 2 - 8 } DASS-21 - Stress & \multicolumn{3}{c}{ Time 1 } & \multicolumn{7}{c}{ Time 2 } \\
\cline { 2 - 8 } & $M$ & $S D$ & $N$ & 11.5 & 7.6 & 18 \\
\hline Normal & 8.8 & 5.7 & 18 & $13.9^{*}$ & 7.3 & 14 \\
Moderate & 20.0 & 2.8 & 14 & $15.8^{* *}$ & 5.5 & 11 \\
Severe+ & 30.4 & 4.4 & 11 & & &
\end{tabular}

Note: ${ }^{*} p<.005 ;{ }^{* *} p<.001$.

course. We encountered the largest effect sizes for the severe groups, where the decrease was notably higher than for the moderate groups. Although due to known methodological issues these results should be treated with caution, the robustness of the results indicates real changes in affective states after mindfulness training.

\section{Affective Symptoms on the DASS-2 I}

In line with past research findings (for example, Broderick, 2005; Miller et al., 1995; Mogg \& Bradley, 2005; Teasdale et al., 1995) we expected that a 10-week meditation course will reduce preexisting levels of depression, anxiety, and stress. Furthermore we explored whether people with moderate and severe levels of affective states responded differently to mindfulness meditation. Our initial hypotheses were confirmed and participants demonstrated an overall decrease within all three affective states. Severe levels showed the most dramatic reductions, whereas moderate levels of anxiety and stress decreased to a smaller extent and moderate depression level remained the same. The hypotheses we put forward were mainly based on increased awareness and detachment from adverse emotional states after attending a meditation course, leading to improvement in affective experiences.

Increased awareness of cognitive and emotional states is at the core of reducing distress (Baer, 2003). By accepting thoughts, emotions, and sensations as they occur, people reduce their roles in maintaining various affective disorders and improve self-management abilities (Roth \& Robbins, 2004; Teasdale et al., 1995). Hence, the first step to reduce affective symptoms through meditation practice is awareness, followed by acceptance and the development of nonjudgmental attitudes. In our results, all affective states showed a decrease in their severity over time, and thus indicate that the presumed increase in awareness has positively influenced our participants' emotional states.

Not all severity levels, however, responded to mindfulness meditation practice the same way. Severe affective states showed a more dramatic decrease than moderate emotional difficulties. In addition, within depression we observed no change for the moderate group and their depression scores remained stable after the meditation course. We propose a motivational explanation for this result.

We suggest that individuals with severe affective ratings may have been more motivated to change. Based on the initial severity levels, a slight increase in awareness and acceptance would have resulted in substantial effects for the severely impaired groups. Therefore, it is highly likely that elevated levels of motivation to change, coupled with the beneficial effects of meditation, have played a significant role in reducing the severe affective states. This assumption is more pronounced 
upon close examination of the results for the severe groups at Time 2. Affective ratings for the severe groups only decreased to moderate levels, and individuals still demonstrated impaired levels of depression, anxiety, and stress. Thus, the large statistical differences did not translate to clinical significance. Rather, our data suggests that while severely depressed, anxious, and stressed individuals clearly benefit from mindfulness training, it is not an adequate treatment modality for severely impaired individuals to achieve normal functioning. On the contrary, people with moderate levels of anxiety and stress may benefit from mindfulness meditation to an extent that they can achieve normal functioning.

Apart from attentional retraining and cognitive restructuring, meditation exerts its effect through various 'byproducts', such as relaxation and self management (Baer, 2003). As anxiety states and stress are associated with increased arousal, tension, and various other physiological symptoms (Marks, 2000; Mogg \& Bradley, 2005), it is not surprising that relaxation, achieved through meditation practices, may have reduced anxiety and stress symptoms for all severity groups, but did not affect moderately depressed individuals. Mindfulness meditation for depression primarily works by initiating cognitive change in clients and is often combined with other cognitive techniques, as in MBCT (Segal et al., 2002). The rationale of using mindfulness techniques within MBCT is not immediate symptom relief, but relapse prevention. Therefore cognitive changes in depression may not be adequately tested immediately after completing a meditation program, but more accurately reflect long term effects after a follow-up.

\section{Limitations of the Present Study and Methodological Issues}

One of the most often mentioned criticisms of mindfulness meditation research is the lack of methodological rigor (Baer, 2003). Unfortunately, our research also suffered from number of methodological limitations.

First and foremost, the lack of a comparison group poses a serious threat to the study's internal and external validity. The lack of control group means that we could not compare the results of the meditation sample with an untreated group, and consequently our statistical conclusion validity should be considered low. Second, our significant results for the DASS-21 measures may have been partially influenced by statistical regression. Although we controlled for regression by incorporating a stable measure into our analyses, the posttest scores of the normal groups within the anxiety and stress conditions showed a slight increase. On the contrary, the severe groups showed an unusually steep decrease in posttest scores and unusually high effect sizes. Thus, extremely low and extremely high pretest scores had a clear tendency to regress to the mean at Time 2 , and it is likely that pretest scores of the normal groups were depressed, whereas the pretest scores of the severe groups were initially inflated by statistical error.

Three factors that protect our results against statistical regression are: the reliability of the DASS-21; the inclusion of a covariate; and the overall robustness of our findings. First, the magnitude of statistical regression is highly dependent on the test-retest reliability of the chosen measure. The higher the reliability, the less the regression will occur. The test-retest reliability of the DASS-21 is high, with .71 for depression, .79 for anxiety, and .81 for stress (Brown, Chorpita, Korotitsch, \& Barlow, 1997). Second, our selected covariate was statistically independent from the dependent measures and showed reliable stability over time. Thus, the inclusion of a covariate into our overall analysis suggests that pretest and posttest scores on the 
DASS-21 reflect real change over time. Third, whereas the large effect sizes for the severe groups may reflect regression, the increase from pretest to posttest for the normal groups did not approach statistical significance. In fact, respondents within the normal depression group demonstrated a slight, but further decrease from Time 1 to Time 2, indicating no regression.

Finally, a number of extraneous variables, for which we did not control for, may have significantly correlated with our posttest measures. Participants may have experienced life events that positively impacted on their emotional states; they may have received other treatment (for example, increased physical exercise) or medication, or received help from other sources. As no information was collected for possible life events, it is difficult to infer that the observed changes in affective states were exclusively related to the practice of mindfulness meditation. Unfortunately, due to time constraints at the times of data collection, no personal information regarding life changes were collected.

Taken together, there are several methodological limitations within the present study that need to be addressed. The lack of control group design, statistical regression, and extraneous variables all adversely influence our internal and external validity. While these limitations may restrict the extent of which we can generalise from the current findings, the magnitude of the results suggests that mindfulness training has a positive effect on the emotional states of depression, anxiety and stress, and deserves a place in treatment considerations. Therefore, the current results should be regarded as an indication of how mindfulness training may aid the treatment of affective disorders and future research, using community samples, should take into consideration the limitations mentioned herein.

\section{References}

Astin, J.A. (2004). Mind-body therapies for the management of pain. Clinical Journal of Pain, 20(1), 27-32.

Baer, R.A. (2003). Mindfulness training as a clinical intervention: A conceptual and empirical review. Clinical Psychology: Science and Practice, 10(2), 125-143.

Barlow, D.H. (1991). Disorders of emotion. Psychological Inquiry, 2(1), 58-71.

Baron-Cohen, \& S., Jolliffe, T. (1997). Is there a 'language of the eyes'? Evidence from normal adults, and adults with autism or Asperger syndrome. Visual Cognition, 4(3), 311-331.

Beck, A.T., \& Clark, D.A. (1988). Anxiety and depression: An information processing perspective. Anxiety Research, 1, 23-36.

Bishop, S.R. (2002). What do we really know about Mindfulness-Based Stress Reduction? Psychosomatic Medicine, 64(1), 71-83.

Bögels, S.M. (2006). Task concentration training versus applied relaxation, in combination with cognitive therapy, for social phobia patients with fear of blushing, trembling, and sweating. Behaviour Research and Therapy, 44(8), 1199-1210.

Bögels, S.M., \& Mansell, W. (2004). Attention processes in the maintenance and treatment of social phobia: Hypervigilance, avoidance and self-focused attention. Clinical Psychology Review, 24(7), 827-856.

Broderick, P.C. (2005). Mindfulness and coping with dysphoric mood: Contrasts with rumination and distraction. Cognitive Therapy and Research, 29(5), 501-510.

Brown, T.A., Chorpita, B.F., Korotitsch, W., \& Barlow, D.H. (1997). Psychometric properties of the Depression Anxiety Stress Scales (DASS) in clinical samples. Behaviour Research and Therapy, 35(1), 79-89.

Brown, K.W., \& Ryan, R.M. (2003). The benefits of being present: Mindfulness and its role in psychological well-being. Journal of Personality and Social Psychology, 84(4), 822-848. 
Grossmana, P., Niemannb, P., Schmidtc, S., \& Walach, H. (2004). Mindfulness-based stress reduction and health benefits: A meta-analysis. Journal of Psychosomatic Research, 57, 35-43.

Hayes, S.C.W., \& Kelly, G. (2003). Mindfulness: Method and process. Clinical Psychology: Science and Practice, 10(2), 161-165.

Hayes, S.C., Strosahl, K., \& Wilson, K.G. (1999). Acceptance and commitment therapy. New York: Guilford Press.

Hunt, C., Keogh, E., \& French, C.C. (2006). Anxiety sensitivity: The role of conscious awareness and selective attentional bias to physical threat. Emotion, 6(3), 418-428.

Kabat-Zinn, J. (1990). Full catastrophe living: The program of the Stress Reduction Clinic at the University of Massachusetts Medical Center. New York: Dell Publishing.

Kabat-Zinn, J. (2003). Mindfulness-based interventions in context: Past, present, and future. Clinical Psychology: Science and Practice, 10(2), 144-56.

Kristeller, J.L., \& Hallett, C.B. (1999). An exploratory study of a meditation-based intervention for binge eating disorder. Journal of Health Psychology, 4, 357-363.

Lovibond, S.H., \& Lovibond, P.E. (1995). Manual for the Depression Anxiety Stress Scales. Sydney, Australia: Psychology Foundation.

Ma, S.H., \& Teasdale, J.D. (2004). Mindfulness-based cognitive therapy for depression: Replication and exploration of differential relapse prevention effects. Journal of Consulting and Clinical Psychology, 72(1), 31-40.

Marks, I.D. (2000). Fear reduction by psychotherapies: Recent findings, future directions. British Journal of Psychiatry, 176, 507-511.

Mogg, K., \& Bradley, B.P. (2005). Attentional bias in generalized anxiety disorder versus depressive disorder. Cognitive Therapy and Research, 29(1), 29-45.

Miller, J.J., Fletcher, K., Kabat-Zinn, J. (1995). Three-year follow-up and clinical implications of a mindfulness meditation-based stress reduction intervention in the treatment of anxiety disorders. General Hospital Psychiatry, 17(3), 192-200.

Nolen-Hoeksema, S. (1991). Responses to depression and their effects on the duration of depressive episodes. Journal of Abnormal Psychology, 100, 569-582.

Nolen-Hoeksema, S. (2000). The role of rumination in depressive disorders and mixed anxiety/depressive symptoms. Journal of Abnormal Psychology, 109(3), 504-511.

Ramel, W.G., Philippe R., Carmona, P.E, \& McQuaid, J.R. (2004). The effects of mindfulness meditation on cognitive processes and affect in patients with past depression. Cognitive Therapy and Research, 28(4), 433-455.

Roth, B., \& Robbins, D. (2004). Mindfulness-based stress reduction and health-related quality of life: Findings from a bilingual inner-city patient population. Psychosomatic Medicine, 66(1), $113-123$.

Salsman, N.L., \& Linehan, M.M. (2006). Dialectical-behavioral therapy for borderline personality disorder. Primary Psychiatry, 13(5), 51-58.

Segal, Z.V., Williams, J.M.G., \& Teasdale, J.D. (2002). Mindfulness-based cognitive therapy for depression: A new approach to preventing relapse. New York: Guilford.

Shapiro, S.L., Astin, J.A., Bishop, S.R., \& Cordova, M. (2005). Mindfulness-based stress reduction for health care professionals: Results from a randomized trial. International Journal of Stress Management, 12(2), 164-176.

Sharma, M.P.K., Mishra, H., \& Balodhi, J.P. (1990). Therapeutic effects of Vipassana Meditation in tension headache. Journal of Personality and Clinical Studies, 6(2), 201-206.

Speca, M., Carlson, L.E., Goodey, E., \& Angen, M. (2000). A randomized, wait-list controlled clinical trial: The effect of a mindfulness meditation-based stress reduction program on mood and symptoms of stress in cancer outpatients. Psychosomatic Medicine, 62(5), 613-622.

Teasdale, J.D. (1999). Emotional processing, three modes of mind and the prevention of relapse in depression. Behaviour Research and Therapy, 37(1), 53-77.

Teasdale, J.D., Segal, Z., \& Williams, J.M.G. (1995). How does cognitive therapy prevent depressive relapse and why should attentional control (mindfulness) training help? Behaviour Research and Therapy, 33(1), 25-39. 
Copyright of Behaviour Change is the property of Australian Academic Press and its content may not be copied or emailed to multiple sites or posted to a listserv without the copyright holder's express written permission. However, users may print, download, or email articles for individual use. 